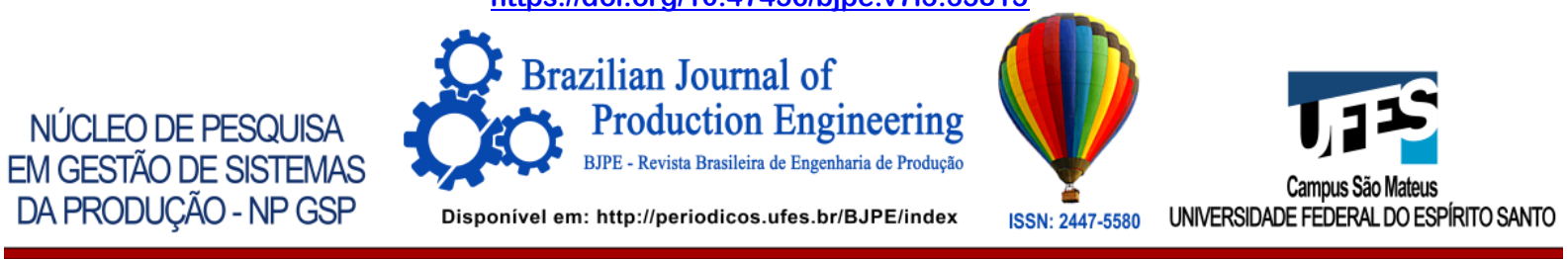

ARTIGO ORIGINAL

OPEN ACCESS

\title{
GESTÃO DE PROJETOS EM EMPRESA DO SETOR AERONÁUTICO NO ESTADO DE SÃO PAULO - BRASIL
}

\section{PROJECT MANAGEMENT IN AN AERONAUTICAL SECTOR COMPANY IN THE STATE OF SÃO PAULO - BRAZIL}

\section{$\underline{\text { Julio Cesar Pinheiro de Santana }}^{1}, \& \underline{\text { Manoel Goncales Filho }}^{2}$ \\ 12 PECEGE - ESALQ - USP, Brasil. \\ $1 *$ engenheiro.jsantana@gmail.com ${ }^{2}$ manoel.goncales01@ fatec.sp.gov.br}

\section{ARTIGO INFO.}

Recebido em: 21.06.2021

Aprovado em: 27.09.2021

Disponibilizado em: 29.09.2021

Palavras-chave:

Análise de Requisitos; Engenharia de Sistemas; Gerenciamento de Escopo.

\section{KEYWORDS:}

Requirements Analysis; Systems engineering; Scope Management.

*Autor Correspondente: Santana, J. C. P.

\section{RESUMO}

O nível de complexidade de um projeto requer atenção especial aos conceitos, ferramentas, etapas e abordagens que devem ser utilizadas para o atendimento das necessidades do cliente. Esse trabalho tem como objetivo identificar projetos realizados na indústria do setor aeronáutico explorando as ferramentas e conceitos que norteiam o projeto para o atendimento aos requisitos do cliente. Uma segunda motivação está em identificar na literatura modelos teóricos que possam suportar o gerenciamento de projetos de alta complexidade, e analisar comparativamente às práticas empregadas nos projetos do setor. Investigou-se uma empresa do setor por meio de uma pesquisa de natureza aplicada, utilizou-se o método comparativo e abordagem qualitativa e o procedimento técnico empregado foi o estudo de caso. Os resultados desta pesquisa trouxeram esclarecimentos às práticas adotadas pela empresa, tais como: o processo de avaliação da real necessidade de um projeto, o entendimento da clareza das necessidades do cliente somado as ações tomadas em cenários de requisitos subjetivos e as interfaces que o gerenciamento de requisitos possui com o gerenciamento de escopo. Além de embasar as respostas dos entrevistados pelos conceitos encontrados na literatura, os resultados contribuíram para a empresa e o setor aeronáutico compreendendo as fases de desenvolvimento e definição das verificações e validações de requisitos necessários a condução do projeto ao atendimento das reais necessidades do cliente.

\begin{abstract}
The level of complexity of a project requires special attention to the concepts, tools, steps and approaches that must be used to meet the client's needs. This work aims to identify projects carried out in the aeronautical industry, exploring the tools and concepts that guide the project to meet customer requirements. A second motivation is to identify theoretical models in the literature that can support the management of highly complex projects, and analyze them comparatively to the practices employed in the sector's projects. A company in the sector was investigated through an applied research, the comparative method and qualitative approach were used and the technical procedure used was the case study. The results of this research brought clarification to the practices adopted by the company, such as: the process of evaluating the real need for a project, understanding the clarity of the customer's needs plus the actions taken in subjective requirements scenarios and the interfaces that the management of requirements have with scope management. In addition to supporting the interviewees' answers by the concepts found in the literature, the results contributed to the company and the aeronautical sector understanding the phases of development and definition of verifications and validations of requirements necessary to conduct the project to meet the real needs of the customer.
\end{abstract}




\section{INTRODUÇÃO}

O desenvolvimento de sistemas complexos desperta a atenção dos profissionais de diferentes áreas como física, biologia, economia, ecologia e ciências sociais (Ottino, 2004). Oehmen et al. (2015) corroboram que um projeto tem alto nível de complexidade quando existem vários módulos ou subsistemas no desenvolvimento que possuem várias conexões entre as eles, na qual observa-se iterações dinâmicas, e o comportamento produzido como resultado dessas iterações não pode ser explicado como a simples soma das partes (comportamento emergente). Conforme os autores, um projeto simples possui estabilidade nas relações entre as partes que o compõe, e as relações de causa e efeito são facilmente identificadas entre elas, o que difere de um projeto complexo.

A complexidade pode ser identificada no trabalho anterior a fase de iniciação do projeto na qual são feitas avaliações de necessidades, elaboração do estudo de caso e a criação do plano de gerenciamento dos benefícios do projeto. Na fase de iniciação do projeto, o PMBOK (2017) menciona a elaboração do Termo de Abertura do Projeto [TAP], emitido pelo patrocinador, que irá realizar o marco inicial autorizando a existência do Projeto e dando autoridade ao gerente de projeto para utilizar os recursos da organização para as atividades do projeto. Contudo, dependendo da complexidade e das informações conhecidas no momento da iniciação do projeto, a quantidade de informações presentes no TAP pode variar. O processo de planejamento é subsequente a fase de iniciação, sendo caracterizado por diversas atividades ao gerenciamento do projeto, do escopo, do cronograma e dos requisitos do projeto. Todavia, a gestão de requisitos é uma atividade crítica em projetos que demandam muitas interfaces entre módulos e vai determinar o sucesso funcional do que está sendo desenvolvido. Não obstante, a elaboração da Matriz de Rastreabilidade dos Requisitos [MRR] realiza a ligação entre os requisitos de produto e as entregas que os satisfazem. Entretanto, projetos complexos exigem uma gestão de requisitos mais detalhada com procedimentos, ferramentas e recursos que permitam a especificação, o desdobramento, a verificação e o controle dos requisitos de projeto (PMBOK, 2017). Logo, os conceitos da Engenharia de Sistemas [ES] apoiam a gestão dos requisitos e possuem critérios rigorosos apresentando um processo sistemático de análise, desenvolvimento de soluções funcionais, sistemas de controle e de verificação de requisitos (DSMC, 2001).

Quando há um processo sistemático para a captura dos requisitos e para o desdobramento das soluções, o projeto se torna robusto em especificar qual a real necessidade do cliente e o que será entregue ao fim do cronograma. O nível de complexidade de um projeto torna as soluções complexas também, o que requer um plano de verificação de requisitos. A ES sugere um processo de verificação onde o produto percorrerá por fases de revisão dentro do diagrama conhecido como "Modelo V". No diagrama prevê-se o início da verificação com uma Revisão Funcional do Sistema [RFS] avaliando o nível mais alto do produto, ou seja, os requisitos que o sistema (conjunto de módulos ou subsistemas) deverá atender. Logo, o sistema é desmembrado em módulos ou subsistemas, no qual os requisitos iniciais de sistema serão desdobrados para os de nível menor, sendo realizada uma Revisão Preliminar do Projeto [RPP]. Descendo mais um nível, todos os requisitos do projeto estarão especificados e serão congelados assim que a Revisão Crítica do Projeto [RCP] for aprovada. Nas próximas etapas,

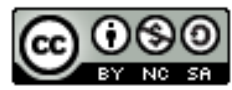


a verificação inicia-se a subida de nível com a fase de fabricação, integração e testes na qual será aplicada a Revisão de Prontidão e Teste [RPT]. Para terminar, o processo de verificação de requisitos é finalizado com a Revisão de Verificação do Sistema [RVS] (DSMC, 2001).

Halligan (2021) propôs uma derivação do "Modelo V" nomeando de "The Wedge Model", na qual os conceitos de verificação e validação são discutidos de maneira mais aprofundada e aplicados ao diagrama. A "Verificação" pode ser definida como uma confirmação por meio de testes e pelo fornecimento de evidências específicas de que os requisitos foram atendidos. A "Validação" pode ser definida como uma confirmação por meio do fornecimento de evidências objetivas de que o uso pretendido especificado para o produto em questão foi realizado no ambiente pretendido. Portanto, utilizando os conceitos de Verificação e Validação, pode-se adicionar diversos processos intermediários de validação de requisitos entre as fases como RFS, RPP, RCP, RPT e RVS trabalhando em conjunto com os processos de verificação já estabelecidos no "Modelo V". Ambos os processos, trabalhando simultaneamente, contribuem para a melhoria do desenvolvimento evitando retrabalhos, gastos e atrasos desnecessários (Halligan, 2021).

A realização de projetos de melhoria e redução de custos tem sido frequente no cenário atual das empresas e a gestão de projetos necessita de procedimentos como os encontrados na ES, os quais auxiliam a gestão dos requisitos do cliente especificando soluções, realizando verificações e atendendo as necessidades do cliente. Nesse contexto, este trabalho tem como objetivo identificar boas práticas de gerenciamento de projeto na indústria do setor aeronáutico explorando as ferramentas e conceitos que norteiam o projeto para o atendimento aos requisitos do cliente. Uma segunda motivação está em identificar na literatura modelos teóricos que possam suportar o gerenciamento de projetos de alta complexidade, e analisar comparativamente às práticas empregadas nos projetos do setor.

\section{MATERIAIS E MÉTODOS}

Neste capítulo abordam-se sobre a natureza, método, objetivos, tipologia da pesquisa, procedimento técnico, instrumento para a coleta de dados e procedimentos de pesquisa teórica para a análise da prática da empresa em estudo e seus resultados.

A pesquisa conduzida é de natureza aplicada com objetivo de gerar novos conhecimentos com foco em uma realidade local e específica (Paranhos, 2014). A pesquisa investiga uma empresa do setor Aeronáutico que apresenta desenvolvimentos de sistemas complexos, ou seja, diversos módulos que possuem interligações entre si com iterações dinâmicas, na qual o comportamento produzido pelo resultado dessas iterações não pode ser explicado somente pela soma das funcionalidades dos módulos, mas sim pela lógica de operação que cada módulo exercita sobre o outro a fim de atingir o objetivo final (Oehmen et al., 2015).

Adotou-se o método comparativo que consiste em investigar os fatos e explicá-los de acordo com as semelhanças e diferenças (Fachin, 2001).

Sob o ponto de vista de seus objetivos a pesquisa é bibliográfica e exploratória. Lakatos e Marconi (2003) relatam quando a pesquisa se encontra na fase preliminar, tem como finalidade proporcionar mais informações advindas do levantamento bibliográfico, entrevistas e análises sobre o assunto que será investigado, na qual possibilita melhor exploração para a 
definição do tema. Segundo as autoras, o objetivo exploratório justifica-se neste tipo de pesquisa, pois levantou-se livros, artigos, teses, dissertações, entre outros, para leitura e exploração dos constructos dos documentos, além das entrevistas e análises sobre o assunto investigado que contribuíram para a discussão dos resultados.

As bases de dados para consulta de livros, teses, dissertações e artigos científicos para a realização desta pesquisa foram: (i) Portal de Periódicos da CAPES; (ii) Biblioteca USP e; (iii) sites específicos da área. As palavras-chave utilizadas nas bases de dados que proporcionaram identificar os documentos para leitura são: (i) Gestão e Análise de Requisitos; (ii) Fases de Desenvolvimento; (iii) Engenharia de Sistemas.

A pesquisa é também explicativa por analisar os fenômenos estudados e identificar causas por meio da interpretação possibilitada pela abordagem qualitativa (Severino, 2000). Possui abordagem qualitativa na qual utilizou-se de dados levantados a campo do ambiente natural, e o pesquisador disposto como agente de interface interpretando significado aos dados coletados (Ludke, 1986).

O procedimento técnico a ser utilizado é o estudo de caso utilizando-se de dados qualitativos de eventos reais com o objetivo de explicar fenômenos ocorridos sob o tema principal desta pesquisa (Eisenhardt, 1989). O protocolo utilizado para as entrevistas será conforme proposto por João Guerra (2010). Segundo o autor, na primeira etapa serão registrados o local, a data da entrevista e os dados do entrevistado (idade, formação acadêmica, função na empresa, setor de atuação, tempo de empresa). Na segunda etapa serão apresentados dados gerais sobre a pesquisa como a organização em estudo, o tipo e a área de pesquisa, o objetivo e as contribuições. Ainda na segunda etapa, de acordo com o autor, será realizado um pré-teste com o objetivo de refinar as questões elaboradas. Na terceira etapa serão apresentadas orientações gerais sobre o questionário que facilitarão as entrevistas. Na quarta etapa serão apresentados os termos técnicos e acrônimos do questionário para a consulta do entrevistado. $\mathrm{Na}$ quinta e última etapa serão realizadas as entrevistas semiestruturadas com questões abertas, e estará disponível um campo para comentários gerais e anotações das discussões relevantes para a pesquisa.

A unidade de análise é uma empresa do ramo Aeronáutico atuando na fabricação de peças que formam o estabilizador horizontal e o cone de cauda de aeronaves feitas em materiais compósitos (fibra de carbono / vidro com resina epóxi) (Tabela 1).

Tabela 1. Perfil da unidade de análise

\begin{tabular}{cccc}
\hline Instituição & $\mathrm{ABC}($ nome fictício) & Sede & Sevilla, Espanha \\
\hline Tempo de Mercado & 15 anos & Setor de Atuação & Aeronáutico \\
\hline Principais Clientes & AIRBUS,BOEING, EMBRAER & Capital & Internacional \\
\hline
\end{tabular}

Fonte: Autores (2021).

A ABC (nome fictício) possui abrangência internacional fornecendo peças para AIRBUS, BOEING e EMBRAER. Ao longo dos 15 anos, a empresa adquiriu conhecimento na fabricação de peças em materiais compósitos para o setor Aeronáutico que exige, minimamente, melhorias em controle, rastreabilidade e gestão de documentações, o que leva a empresa a desenvolver projetos de melhorias e adequações das suas instalações. Sendo que as 
Citação (APA): Santana, J. C. P., \& Gonçales, M., Filho (2021). Gestão de projetos em empresa do setor aeronáutico no estado de São Paulo - Brasil. Brazilian Journal of Production Engineering, 7(3), 219-233.

entrevistas semiestruturadas ocorreram com gerentes e engenheiros da planta considerados "best expert" que possuem experiência em gestão de projetos (Tabela 2).

Tabela 1. Perfis dos entrevistados - amostras da pesquisa

\begin{tabular}{ccccccc}
\hline Entrevistado & Sexo & Idade & Formação & Cargo & $\begin{array}{c}\text { Tempo de } \\
\text { Experiência } \\
\text { na Função }\end{array}$ & $\begin{array}{c}\text { Tempo } \\
\text { de } \\
\text { Empresa }\end{array}$ \\
\hline 01 & Masculino & 50 & $\begin{array}{c}\text { Engenharia Civil/ } \\
\text { MBA Gestão de Projetos }\end{array}$ & Engenheiro Sênior & 30 anos & 6 meses \\
\hline 02 & Masculino & 45 & $\begin{array}{c}\text { Engenharia Mecânica/ } \\
\text { MBA em Gestão } \\
\text { Empresarial }\end{array}$ & $\begin{array}{c}\text { Gerente de } \\
\text { Engenharia de } \\
\text { Manufatura }\end{array}$ & 21 anos & 3 anos \\
\hline
\end{tabular}

Fonte: Autores (2021).

O entrevistado 1 é formado em Engenharia Civil com "Master in Business Administration" [MBA] em Gestão de Projetos com 30 anos de experiência como Engenheiro de Manufatura e seis meses de empresa. O entrevistado 2 é formado em Engenharia Mecânica com MBA em Gestão Empresarial, com 21 anos de experiência e três anos de empresa como gerente de manufatura. Desse modo, após definir a estrutura conceitual teórica, planejar os casos unidades de análise, definir a amostra, conduzir o teste piloto, coletar e analisar dados de projetos realizados pela empresa em estudo, discutiu-se os resultados do estudo de caso comparativamente as implicações teóricas.

\section{RESULTADOS E DiscuSSÃo}

A pesquisa teórica e a identificação das contribuições da literatura embasaram os resultados práticos desta pesquisa. De acordo com as contribuições dos autores, que suportam os temas principais abordados na direção esperada dos objetivos desta pesquisa, foram considerados na construção e análise da discussão dos resultados deste estudo de caso. Nesse contexto, a partir da Tabela 3 foram apresentadas as implicações teóricas, de modo abrangente e relevante, do que foi utilizado do levantamento feito da revisão teórica no estudo de caso. Todavia, na sequência foram detalhadas as contribuições dos autores, com os achados das entrevistas de campo do estudo de caso desta pesquisa.

Tabela 2. Aspectos principais aplicados nos resultados da pesquisa

\begin{tabular}{|c|c|c|}
\hline Temas abordados na pesquisa & $\begin{array}{c}\text { Objetivos de pesquisa e contribuições } \\
\text { dos autores }\end{array}$ & Autores \\
\hline $\begin{array}{l}\text { Definir uma estrutura conceitual } \\
\text { teórica }\end{array}$ & $\begin{array}{c}\text { Identificar na literatura as contribuições } \\
\text { potenciais dos autores para embasar os } \\
\text { resultados desta pesquisa }\end{array}$ & $\begin{array}{c}\text { (Ottino, 2004) } \\
\text { (PMBOK, 2017) } \\
\text { (DSMC, 2001) } \\
\text { (Oehmen et al., 2015) }\end{array}$ \\
\hline $\begin{array}{l}\text { Definir o protocolo, planejar } \\
\text { teste piloto para o questionário e } \\
\text { examinar uma unidade de } \\
\text { análise do setor Aeronáutico }\end{array}$ & $\begin{array}{l}\text { Identificar quais são os achados na } \\
\text { literatura para investigar às práticas de } \\
\text { projetos na unidade de análise }\end{array}$ & $\begin{array}{c}\text { (João Guerra, 2010) } \\
\text { (Eisenhardt, 1989) } \\
\text { (Ludke, 1986) } \\
\text { (Severino, 2000) } \\
\text { (Fachin, 2001) } \\
\text { (Paranhos, 2014) }\end{array}$ \\
\hline $\begin{array}{l}\text { Conduzir entrevistas } \\
\text { semiestruturadas e identificar o } \\
\text { estado atual da unidade de } \\
\text { análise do setor Aeronáutico }\end{array}$ & $\begin{array}{l}\text { Identificar o estado atual e futuro de } \\
\text { gestão de projetos }\end{array}$ & (PMBOK, 2017) \\
\hline Definir a Gestão de Requisitos & $\begin{array}{l}\text { Verificar como a Gestão de Requisitos é } \\
\text { entendida pela unidade e como ela se } \\
\text { aplica na Gestão de Projetos }\end{array}$ & $\begin{array}{l}\text { (PMBOK, 2017) } \\
(\mathrm{DSMC}, 2001)\end{array}$ \\
\hline
\end{tabular}

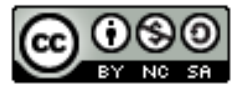



Brasil. Brazilian Journal of Production Engineering, 7(3), 219-233.

\begin{tabular}{|c|c|c|}
\hline Temas abordados na pesquisa & $\begin{array}{l}\text { Objetivos de pesquisa e contribuições } \\
\text { dos autores }\end{array}$ & Autores \\
\hline $\begin{array}{l}\text { Definir os impactos da falta de } \\
\text { Gestão de Requisitos }\end{array}$ & $\begin{array}{l}\text { Identificar quais são os impactos que a } \\
\text { falta de Gestão de Requisitos pode } \\
\text { ocasionar em um Projeto }\end{array}$ & $\begin{array}{l}\text { (PMBOK, 2017) } \\
(\text { FAB, 2021) }\end{array}$ \\
\hline $\begin{array}{l}\text { Definir fases do projeto em que } \\
\text { os requisitos devem ser } \\
\text { verificados }\end{array}$ & $\begin{array}{c}\text { Identificar quais são as fases de um } \\
\text { projeto e quais delas os requisitos devem } \\
\text { ser verificados. }\end{array}$ & $\begin{array}{c}\text { (PMBOK, 2017) } \\
\text { (DSMC, 2001) } \\
\text { (Graessler } \text { et al., 2018) } \\
\quad \text { (Halligan, 2021) } \\
\text { (Olechowski et al., 2015) }\end{array}$ \\
\hline $\begin{array}{l}\text { Definir como a Gestão de } \\
\text { Requisitos se acomoda em } \\
\text { diferentes abordagens de } \\
\text { projetos }\end{array}$ & $\begin{array}{l}\text { Identificar como as ferramentas, recursos } \\
\text { e fases de verificação de requisitos se } \\
\text { aplicam aos projetos com abordagem } \\
\text { preditiva, adaptativa e híbridos. }\end{array}$ & $\begin{array}{c}\text { (PMBOK, 2017) } \\
\text { (DSMC, 2001) } \\
\text { (Graessler et al., 2018) } \\
\text { (Halligan, 2021) } \\
\text { (Olechowski } \text { et al., 2015) }\end{array}$ \\
\hline $\begin{array}{l}\text { Definir quais são os impactos de } \\
\text { mudanças de requisitos }\end{array}$ & $\begin{array}{l}\text { Identificar os impactos que uma } \\
\text { solicitação de mudança de requisitos pode } \\
\text { ocasionar em um projeto e quais boas } \\
\text { práticas devem ser tomadas. }\end{array}$ & $\begin{array}{c}\text { (PMBOK, 2017) } \\
\text { (Oehmen et al., 2015) }\end{array}$ \\
\hline $\begin{array}{l}\text { Definir as responsabilidades de } \\
\text { profissionais focados em Gestão } \\
\text { de Requisitos }\end{array}$ & $\begin{array}{l}\text { Identificar quais são as responsabilidades, } \\
\text { e como poderia ser arquitetado um time } \\
\text { de Engenharia de Sistemas. }\end{array}$ & (PMBOK, 2017) \\
\hline $\begin{array}{l}\text { Propor um estado futuro com } \\
\text { redução de custos }\end{array}$ & Gestão de requisitos do cliente & $\begin{array}{c}\text { (PMBOK, 2017) } \\
\text { (DSMC, 2001) } \\
\text { (Graessler } \text { et al., 2018) } \\
\text { (Halligan, 2021) } \\
\text { (Olechowski et al., 2015) }\end{array}$ \\
\hline
\end{tabular}

As características dos temas abordados, os objetivos e contribuições dos autores e as etapas identificadas na literatura foram consideradas nesta pesquisa, sendo apresentadas nos parágrafos a seguir.

\section{Definição da Gestão de Requisitos}

A respeito do entendimento do que é de fato a Gestão de Requisitos, o entrevistado 1 descreve que pode ser entendida como um conjunto de diretrizes e procedimentos que definem requisitos de projeto que caracterizam um produto, como por exemplo, requisitos funcionais, de performance, autonomia e vida útil. $\mathrm{O}$ entrevistado 2 declara que a gestão de requisitos é um mapeamento do desejo do cliente, na qual busca entender seus desejos para traduzir em uma linguagem técnica e específica. O PMBOK (2017) descreve que o pilar de gerenciamento da qualidade do projeto contempla a organização, gerenciamento e controle de requisitos de qualidade do projeto e do produto, visando atender as expectativas dos "stakeholders" - partes interessadas. $\mathrm{O}$ entrevistado 2 relata que na empresa existem projetos que se iniciam com requisitos técnicos bem determinados, assim como existem outros projetos que necessitam desenvolver os requisitos até a maturidade, para um controle da necessidade do cliente, desde o ponto em que ela é subjetiva, até chegar em definições objetivas.

De acordo com DSMC (2001) um processo de engenharia de sistemas está apresentado pela Figura 1 na qual a análise de requisitos está contida. No processo as entradas abrangem as necessidades, objetivos e requisitos do cliente somados a uma série de outros fatores. Desse modo, inicia-se o processo de análise de requisitos, baseando-se na missão e no ambiente do projeto, identificando os requisitos funcionais, definindo os requisitos de desempenho e as restrições de projeto. Em seguida, esses requisitos são desdobrados para níveis inferiores 
desenvolvendo soluções funcionais, alocando os requisitos de desempenho e as limitações de projeto para todo os níveis funcionais, definindo e refinando as interfaces internas e externas e a arquitetura funcional do projeto.

Entretanto, é previsto um "loop" entre o processo de análise de requisitos e o de análise e solução funcional, pois cada projeto poderá ter uma necessidade própria em revisar, readequar, reescrever ou corrigir os requisitos capturados no início do projeto. O terceiro nível é definido como processo de síntese do projeto na qual ocorre a transformação das arquiteturas, ou seja, o projeto começa a se materializar definindo itens de configuração, elementos de sistemas, selecionando as soluções, refinando as interfaces físicas internas e externas e definindo conceitos alternativos para o sistema. Esses três processos são suportados o tempo todo por um processo de análise e controle do sistema responsável por estudos de "trade-off' (estudos que examinam alternativas viáveis para definir qual é o melhor caminho a ser seguido), análise de eficácia, gerenciamento de riscos, configurações, interface, dados e medição de desempenho. As saídas do processo são principalmente o item de arquitetura do sistema, o avanço nos níveis de desenvolvimento do projeto e a definição de linhas de base. Portanto, um dos resultados desta pesquisa foi a identificação da possibilidade de aplicação / utilização de uma ferramenta da Engenharia de Sistemas para o apoio na gestão de requisitos.

Figura 1. O processo de Engenharia de Sistemas

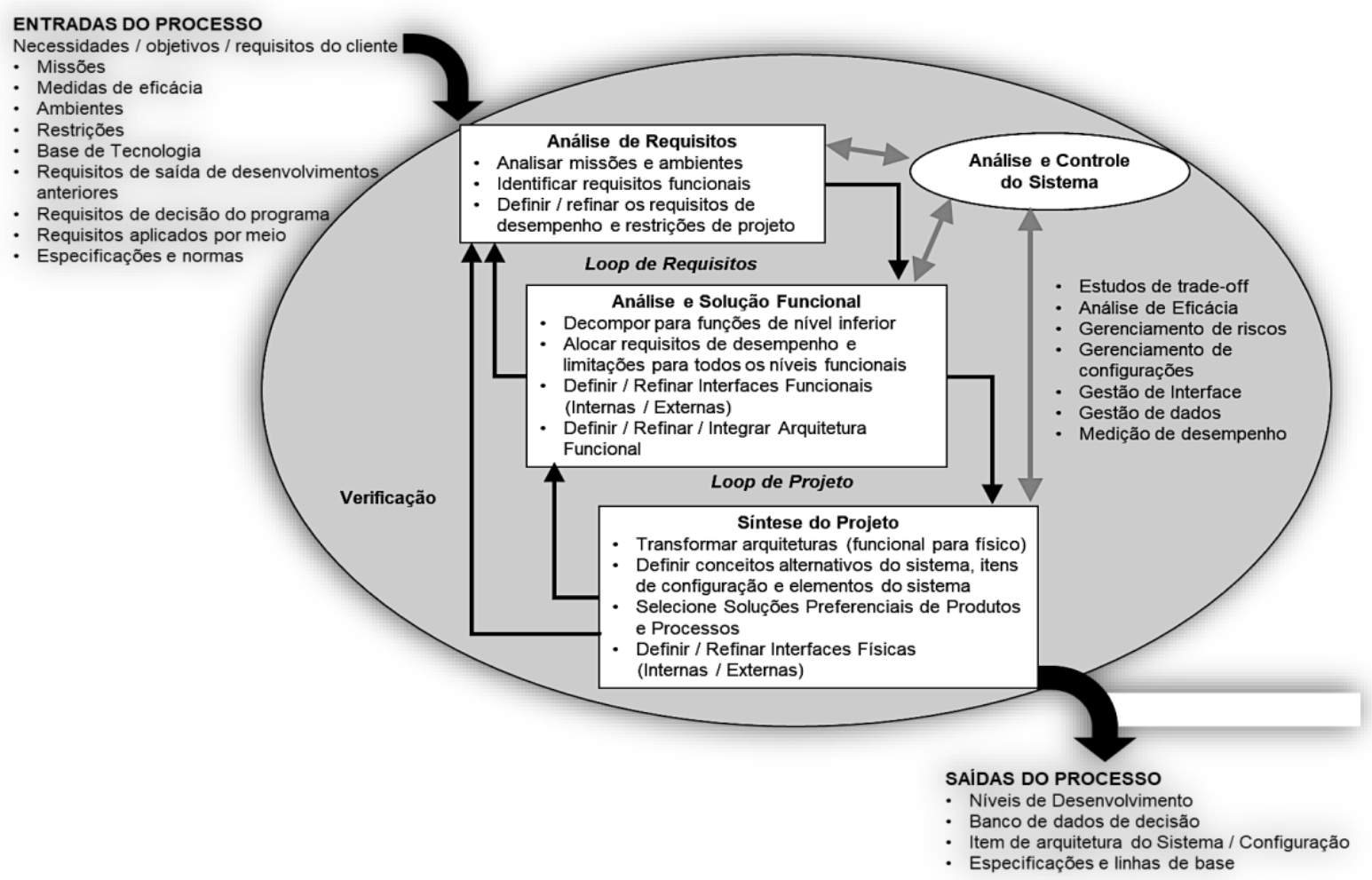

Fonte: DSMC (2001)

\section{Impactos da Falta de Gestão de Requisitos}

Abordou-se sobre os impactos que a falta de gestão de requisitos pode gerar em um projeto, e de acordo com a experiência do entrevistado 1, o impacto mais comum é o retrabalho ou a reinicialização de um projeto. $\mathrm{O}$ entrevistado aponta com base em sua experiência em 
desenvolvimento de aeronaves, os requisitos de peso, custo e de durabilidade são os mais críticos e podem afetar um projeto ao ponto de descartar anos de trabalho. O entrevistado 2 relata que em um cenário de desenvolvimento de "softwares", a falta de gestão de requisitos pode resultar em um produto cheio de erros, lentidão, travamentos e saturação de servidores de Tecnologia da Informação [TI]. Esse cenário pode ser evitado se os requisitos de desempenho forem bem escritos, os investimentos em infraestrutura sejam compatíveis com o que o produto irá demandar, e ainda os requisitos de interface entre módulos sejam claros respeitando os limites de "hardware".

Nesse contexto, de acordo com PMBOK (2017), o custo da qualidade [CDQ] incluí o não cumprimento de requisitos que gera retrabalhos, custos de falhas internas ou externas, também chamados de custos de má qualidade. Não obstante, as falhas externas, ou seja, aquelas encontradas pelo cliente, são mais críticas pois envolvem responsabilidades, trabalho de garantia de qualidade, negociações ou até a perda de negócios. Logo, projetos complexos naturalmente vão possuir requisitos complexos, principalmente aqueles que especificam interfaces entre diferentes módulos, e a falta de gestão deles pode resultar na desidratação da imagem da empresa e dos relacionamentos com os "stakeholders" principais conduzindo a organização a declínio.

Entretanto, o entrevistado 1 argumenta que antes mesmo de iniciar a especificação e definição de requisitos, é importante realizar a pesquisa de mercado junto a um estudo de viabilidade econômica ("Business Case"). Após isso, deve-se realizar uma fase de anteprojeto na qual a empresa deverá fazer uma espécie de "rascunho" do produto realizando estimativas de custos recorrentes e não recorrentes, levantando a necessidade de infraestrutura e outros recursos que serão necessários. Essas duas etapas preliminares são tão importantes quanto a especificação de requisitos, pois você pode ter um conjunto de requisitos muito bem elaborado, mas falhar na pesquisa de mercado, ou seja, ter um produto final que atenda aos requisitos, mas que nenhum "stakeholder" tem interesse. Da mesma forma, o entrevistado citou a experiência do desenvolvimento do avião EMBRAER CBA 123 que foi considerado o avião mais moderno do seu tempo, mas que teve o projeto prematuramente cancelado devido aos altos custos de produção (FAB, 2021).

A realização do "business case" é importante para a determinação da validade dos benefícios de um produto do qual não se tem informações suficientes, obtendo os objetivos e um racional para a inicialização de um projeto. A literatura define que uma avaliação de necessidades precede o business case e compreenderá melhor os objetivos, metas, problemas e oportunidades do negócio (PMBOK, 2017). Se essas boas práticas tivessem sido aplicadas ao caso do CBA 123, talvez ocorressem a especificação de requisitos que não iria tornar o avião mais avançado da época, mas que poderia deixar o projeto economicamente viável para o mercado.

\section{Definição das fases do projeto na qual os requisitos devem ser verificados}

Com relação as fases de projeto na qual os requisitos devem ser verificados, o entrevistado 1 relata que elas devem ser claras, descritas por um cronograma que defina quais são os entregáveis ao final de cada fase e quando os requisitos serão verificados e validados. Com 
base em sua experiência, o entrevistado 1 observa que essas fases de projeto eram prédeterminadas no contrato de desenvolvimento com o cliente tendo um cronograma de atividades para ser cumpridas, e a gestão dessas fases era essencial para mostrar o avanço do projeto para os "stakeholders", mesmo que nas fases iniciais de aprofundamento do projeto sejam identificadas necessidades de adaptações e, consequentemente, alguns retrocessos e avanços. Embora foi mencionada as necessidades de adaptações, o entrevistado 1 acredita que grande parte dos projetos vivenciados no setor Aeronáutico possuíram abordagens preditivas. Ele relata que ainda existem riscos quando um projeto possui eventos financeiros atrelados ao término de uma fase, ou seja, quando o não cumprimento de qualquer fase pode gerar uma falta de pagamento por parte do cliente. Nesse cenário, não é raro observar gerentes de projeto com o desejo de terminar uma fase ou marco temporal a qualquer custo, mesmo que isso signifique omitir problemas de desenvolvimento e falta de transparência nas comunicações do andamento do projeto. Assim, é essencial o projeto identificar problemas de desenvolvimento e ter registrado em seu plano de gerenciamento de riscos os apetites por riscos do cliente (principal "stakeholder"), ou seja, os limites dos riscos mensuráveis determinando o nível aceitável de exposição ao risco (PMBOK, 2017).

O entrevistado 2 afirma que se os requisitos forem subjetivos e as necessidades do cliente não estejam claras, torna-se necessário realizar reuniões periódicas com o cliente para que se alcance a maturidade do real escopo do projeto. Portanto, requisitos que não estão claros podem demandar mudanças constantes o que conduz o projeto a uma abordagem adaptativa ou híbrida. Após tornar os requisitos mais claros e objetivos, o projeto deve ser conduzido para o desenvolvimento e consequentemente as fases de verificação. DSMC (2001) corrobora com a divisão de fases proposta pela Engenharia de Sistemas, também conhecido como modelo "V", que se aplica ao desenvolvimento de projetos complexos. As etapas de revisões de projetos são consideradas marcos temporais que definem fases de revisão do projeto (Figura 2).

Figura 2. Engenharia de Sistemas e Verificação

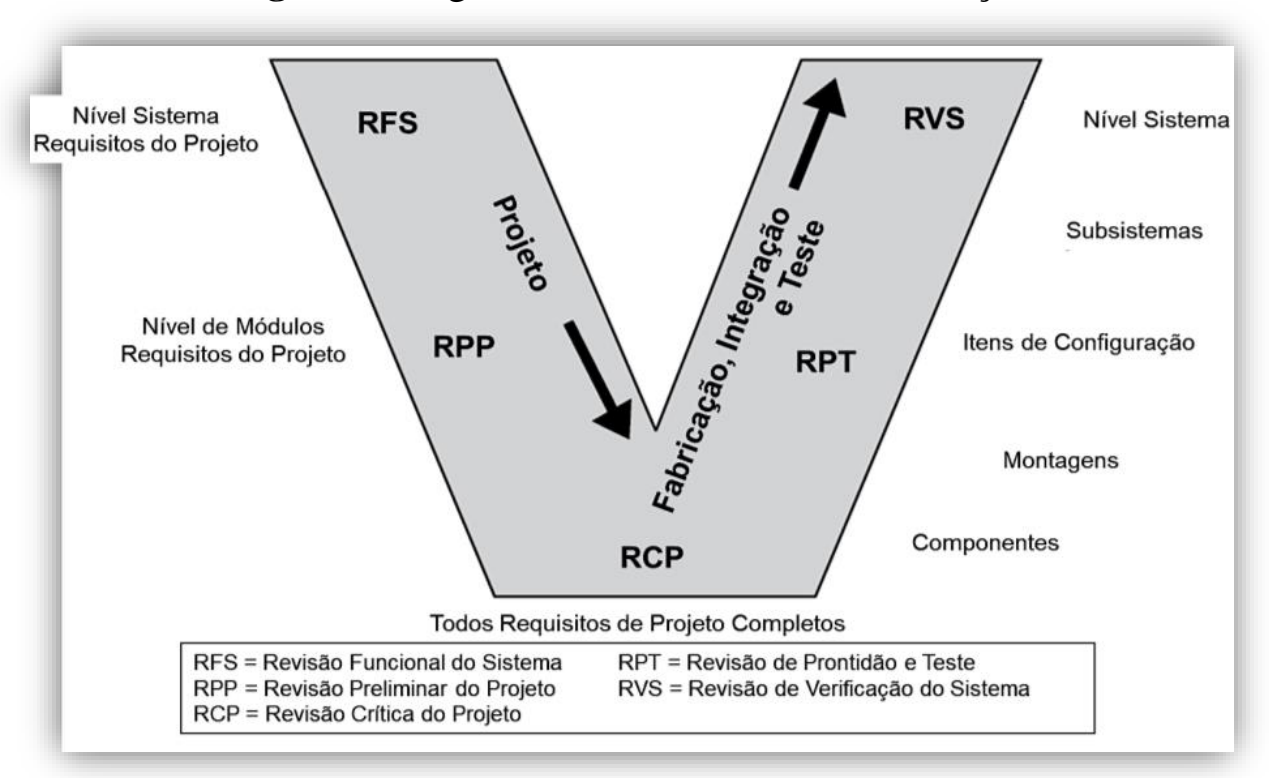

Fonte: DSMC (2001) 
No entanto, Graessler et al. (2018) realizaram um estudo sobre diversas propostas de adaptações do modelo " $\mathrm{V}$ " encontrados na literatura, na qual uma dessas adaptações é conhecida como "modelo V estendido" proveniente do Departamento de Transporte dos Estados Unidos. Nesse modelo, observam-se fases de desenvolvimento do projeto desde a pesquisa de mercado, anteprojeto, "business case", desenvolvimento do produto, verificação e validação, operação e plano de manutenção, mudanças e atualizações, e à obsolescência. Essas fases são enumeradas de -1 até 5 conforme mostra a Figura 3.

Figura 3. Modelo V do Departamento de Transporte dos Estados Unidos.

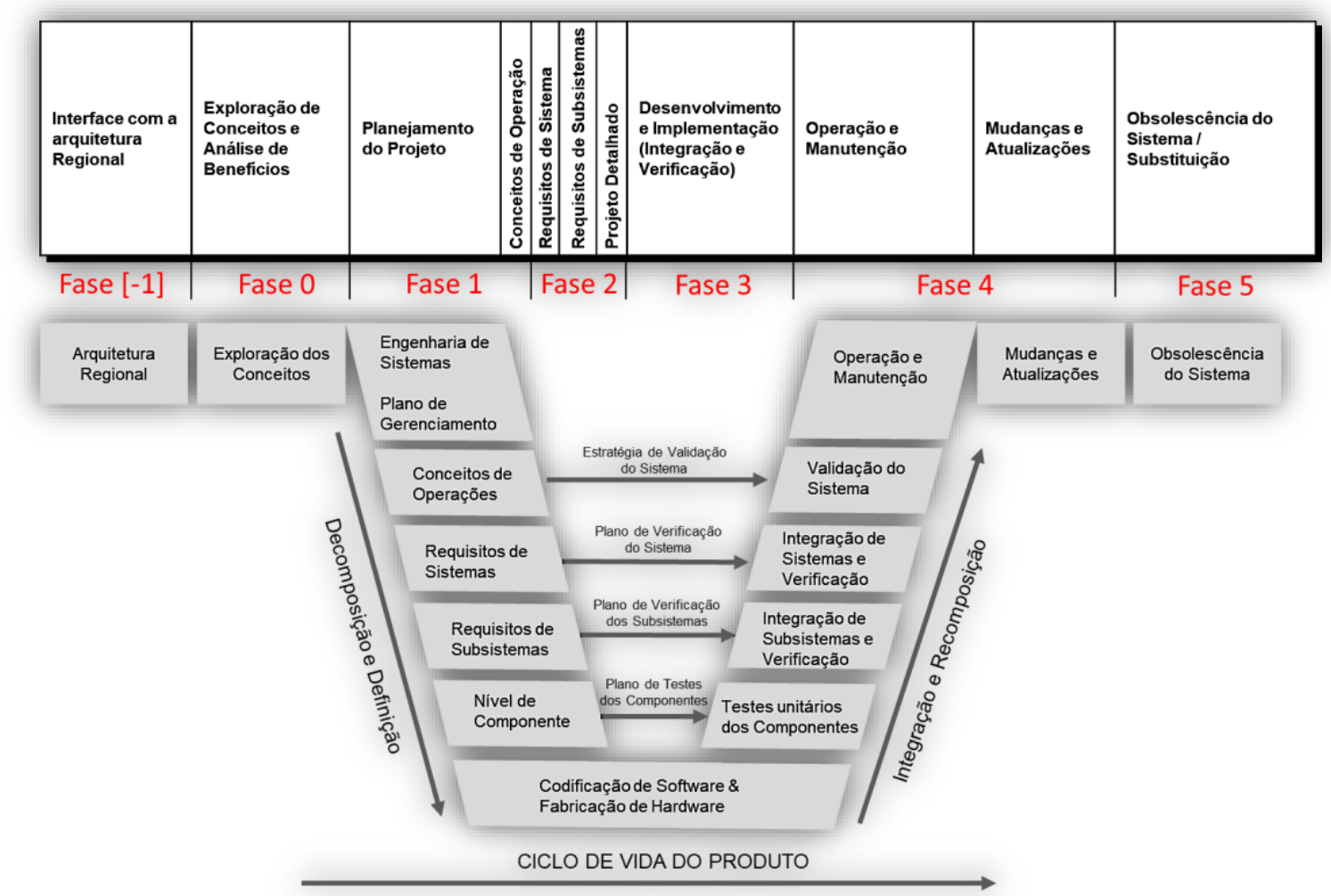

Fonte: Graessler et al. (2018)

As propostas de fases observadas nas Figuras 2 e 3 estão sempre relacionadas a uma decomposição do sistema capturando requisitos na direção "TOP" $\square$ " $D O W N$ " e verificando e validando requisitos em "BOTTOM" $\square$ "UP". De modo geral, ambas propostas interpretam as fases de um projeto de maneira cartesiana regular, ou seja, os requisitos são especificados, a estrutura é decomposta até o nível mais baixo e depois disso inicia-se o processo de verificação, integração e validação do produto. Desta forma, os projetos que possuem uma abordagem preditiva possuem facilidade em se adaptar aos modelos das Figuras 2 e 3, mas não é o caso das abordagens adaptativas, pois os requisitos podem sofrer modificações constantes até que se alcance uma maturidade de projeto. O modelo chamado "The Wedge Model", proposto por Halligan (2021), sugere que ocorram processos cíclicos de verificação e validação não somente na região "BOTTOM" $\rightarrow$ "UP" como também na decomposição do projeto, ou seja, na descida "TOP" $\rightarrow$ "DOWN" gerando um modelo dinâmico de pequenos avanços e retrocessos ao longo do desenvolvimento do projeto. Dessa forma, projetos com abordagens adaptativas possuem uma melhor acomodação ao "The Wedge Model".

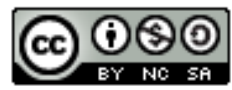


Quando uma metodologia acomoda uma abordagem preditiva e uma outra metodologia acomoda uma abordagem adaptativa, consequentemente, ambas serão úteis para uma abordagem híbrida. Assim, o nível de maturidade dos requisitos do cliente precisa ser mapeado e analisado pois, se forem subjetivos conforme relatado pelo entrevistado 2, uma série de reuniões deveriam ser realizadas com o cliente a fim de tornar o escopo mais claro. Olechowski et al. (2015) apresentaram o uso do sistema conhecido no inglês como "Technology Readiness Level" [TRL] desenvolvido pela "National Aeronautics and Space Administration" [NASA] nos anos 1970 como uma ferramenta para o auxílio de verificação da maturidade de tecnologias durante o desenvolvimento de sistemas complexos. O sistema funciona por meio de um questionário extenso que ao final classificará o nível de maturidade de certa tecnologia (Tabela 4).

Tabela 3. Níveis de TRL

\begin{tabular}{c|c}
\hline Nível TRL & Definição \\
\hline TRL9 & Aprovação do sistema através de campanhas de testes sucessivos \\
TRL8 & Qualificação de campo através de testes e demonstração do sistema final \\
TRL7 & Demonstração do Protótipo em ambiente real \\
TRL6 & Modelo ou protótipo de demonstração em ambiente relevante \\
TRL5 & Validação do componente e/ou equipamento em ambiente relevante \\
TRL4 & Validação do componente e/ou equipamento em ambiente laboratorial \\
TRL3 & Função crítica analítica e experimental e/ou prova do conceito característico \\
TRL2 & Conceito tecnológico e/ou aplicação formulada \\
TRL1 & Os princípios básicos são observados e registrados \\
\hline
\end{tabular}

Fonte: Olechowski et al. (2015)

No cenário de projetos, a classificação TRL pode ser útil para a decisão de qual abordagem será utilizada para o desenvolvimento do projeto, ou seja, classificações próximas a TRL1 expressam requisitos e especificações desconhecidas que consequentemente são propensos à uma abordagem adaptativa e/ou híbrida. Por outro lado, classificações entre TRL7 a TRL9 demonstram requisitos e especificações mais definidos que são propensos a uma abordagem híbrida e/ou preditiva. Olechowski et al. (2015) apresentaram a Figura 4 na qual observa-se os níveis de maturidade em função de cada etapa TRL e também os marcos temporais descritos com as letras A, B e C.

Figura 4. Mapeamento dos níveis de preparação da tecnologia para o processo de aquisição do sistema do Departamento de Defesa dos EUA.

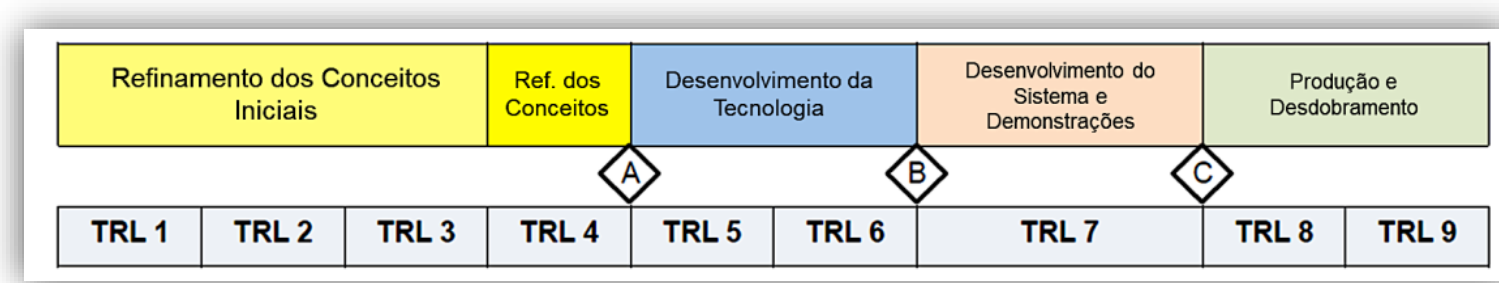

Fonte: Olechowski et al. (2015)

Esses marcos temporais definem uma evolução na maturidade do projeto na qual se observasse que a partir de "B" inicia-se o desenvolvimento do sistema e as demonstrações, verificações e validações de requisitos. Assim, considerando que o projeto começa a ter requisitos mais definidos a partir do marco temporal " $\mathrm{B}$ ", os conceitos de desdobramento de 
requisitos apresentados por DSMC (2001) na Figura 2, e os apresentados por Graessler et al. (2018) na Figura 3, e os descritos por Halligan (2021) podem ser aplicados em conjunto com a classificação TRL apresentada por Olechowski et al. (2015) na Tabela 4. Portanto, encontrou-se uma oportunidade de utilização desses conceitos e ferramentas em projetos complexos para gerenciar as fases de evolução de um projeto ao longo do ciclo de vida.

\section{Definição de quais os impactos de mudanças de requisitos}

No tocante aos impactos que as solicitações de mudanças de requisitos podem causar em um projeto, o entrevistado 1 afirma que as estimativas de custo, recursos e infraestrutura que foram realizadas no início do projeto nem sempre serão exatas e os projetos precisam estar preparados para mudanças, tanto os preditivos quanto os adaptativos. Relatou-se que o plano de gerenciamento de riscos poderá mapear mudanças e possíveis problemas que o projeto poderá ter, e os projetos deverão estar preparados para realizar mudanças sempre que for necessário, principalmente quando envolve a segurança de um produto, mesmo que ocorram impactos nas soluções técnicas que foram definidas.

Existem mudanças que frequentemente ocorrem em projetos complexos, e na literatura está definida como problemas graves ("wicked problems"), conhecidos também como problemas que o cliente é incapaz de apresentar requisitos claros com antecedência até que se experimente as soluções dadas a um protótipo (Oehmen et al., 2015). Desta forma, o projeto sofrerá impacto ao absorver novos requisitos que somente foram mapeados em uma fase posterior ao início de projeto. Na opinião do entrevistado 1, o impacto mais relevante é aquele que não apresenta uma solução imediata e o projeto precisa descobrir qual caminho seguir de forma empírica.

A ocorrência desse cenário pode ser causada por uma tratativa inicial errada, ou seja, quando um projeto complexo recebeu as mesmas tratativas de um projeto simples. Oehmen et al. (2015) adaptaram uma frase de Henry Louis Mencken afirmando que em projetos complexos haverá sempre uma solução caracterizada como simples, clara e errada, e quando essa solução é perseguida, o ambiente do projeto passará de complexo para caótico.

O entrevistado 2 menciona que os projetos que não atingem um escopo definido estarão susceptíveis a entrar em ciclos parasitários que não evoluem o projeto, pois em todo momento está se tendo mutações. Desta forma, se o cenário for de uma requisição de mudança realizada em um projeto com escopo bem definido, a modificação envolverá a análise de impactos, escopo, tempos e possíveis aditivos contratuais. Entretanto, se o cenário for de uma requisição de mudança realizada em um projeto com escopo subjetivo, a tendência é que esse projeto nunca tenha um fim.

$\mathrm{Na}$ visão do entrevistado 2 a solicitação de mudança pode afetar diretamente na gestão de escopo e conduzir a conflitos com o cliente, caso não seja bem gestionada. $\mathrm{O}$ respondente relatou uma experiência em que os requisitos de um projeto de um equipamento industrial foram especificados aquém da real necessidade. Em um determinado momento do projeto, o cliente buscou corrigir esse requisito para os parâmetros corretos por meio de reuniões informais na qual não se teve registro algum do que foi solicitado, ou seja, não houve uma comunicação formal com o desenvolvedor. O equipamento foi entregue conforme a 
especificação inicial e, devido a falha na gestão de mudanças e na gestão de comunicação, ocorreu um conflito entre cliente e desenvolvedor que iniciou discussões de caráter judicial. Por fim, o acordo final envolveu um aditivo contratual por parte do cliente e também uma flexibilização do desenvolvedor na correção desses requisitos.

O PMBOK (2017) corrobora que os conflitos são inevitáveis no desenvolvimento de um projeto e o gerente de projetos precisa ter a capacidade de negociar e influenciar tanto conflitos entre a equipe de projeto quanto conflitos com o cliente. Uma solução comum para um conflito é a definida como situação perde-perde na qual ambas as partes buscam a conciliação em um cenário que forneça um grau de satisfação para todas as partes.

\section{Definição das responsabilidades de profissionais focados em Gestão de Requisitos}

A respeito das responsabilidades e da gestão de requisitos, o entrevistado 1 afirma que é importante se ter um time multidisciplinar trabalhando para dar soluções aos requisitos de projeto, e que são profissionais de diversas áreas como mecânica, elétrica, eletrônica, química, computacional ou outras, de acordo com a área de atuação da empresa. Entretanto, o entrevistado 1 apresenta uma ressalva que existem projetos complexos que demandam um profissional exclusivo para gerir os requisitos técnicos quando o número de requisitos é alto deixando o time multidisciplinar focado nas soluções e o profissional exclusivo fazendo "follow up" sobre o andamento do atendimento aos requisitos. O entrevistado 2 observa que a gestão de requisitos deve ser realizada pelo corpo técnico, mas o gerente de projeto deve ter a responsabilidade de gerir o escopo por meio do conteúdo gerado pelo escopo técnico. Nessa direção, o PMBOK (2017) apresenta o plano de gerenciamento do escopo do projeto como um conjunto de processos, sendo: coletar e gerenciar o conhecimento dos requisitos, realizar a validação e controle, definir o escopo e criar a Estrutura Analítica do Projeto [EAP].

O cumprimento dos requisitos do projeto significa reduzir custos de má qualidade, retrabalhos e atrasos, sendo que a Gestão de Requisitos deve ser realizada pelo pilar de Gerenciamento da Qualidade (PMBOK, 2017). Encontrou-se uma oportunidade no uso das propostas da Engenharia de Sistemas apresentados por DSMC (2001) e Graessler et al. (2018) em paralelo com o uso da classificação TRL apresentada por Olechowski et al. (2015), pois foram observados modelos que suportam a abordagem preditiva, adaptativa e híbrida. Nesse cenário, esses modelos suportam a gestão de requisitos mesmo quando são subjetivos, até alcançarem a clareza e objetividade.

\section{CONCluSÃo}

Foram realizadas entrevistas semiestruturadas com gerentes e engenheiros da empresa $\mathrm{ABC}$ considerados "best expert" em gestão de projetos, com o objetivo de capturar as práticas, conhecimentos e as experiências, e embasá-las às recomendações da literatura. Apesar das contribuições da literatura se apresentarem intercambiáveis aos diferentes setores, a pesquisa foi realizada com o foco na indústria aeronáutica que possui peculiaridades, requisitos, processos e desdobramentos únicos. As experiências dos entrevistados contribuíram com o trabalho pelo esclarecimento e indicação das práticas adotadas no ramo aeronáutico, como: avaliar a real necessidade de um projeto, entender se os requisitos do cliente estão claros, planejar o gerenciamento de requisitos para subsidiar o gerenciamento de escopo e zelar pela 
comunicação formal de mudanças que podem ocorrer no projeto. Identificou-se que o entendimento de fases de desenvolvimento e definição do momento de verificação de requisitos foram assuntos não muito claros dentro do campo de conhecimento dos entrevistados. Nesse ponto, os resultados apresentados na literatura trouxeram contribuições relevantes para a identificação de fases e períodos de verificação e validação de requisitos ao longo do ciclo de desenvolvimento de um projeto. Nesse contexto, os conceitos da Engenharia de Sistemas somados a classificação TRL são recomendados para todo tipo de abordagem de projeto, principalmente os híbridos. Contudo, esta pesquisa tem algumas limitações no método, como à sua população e amostra reduzida e pela subjetividade das respostas, ou seja, as respostas foram frequentemente abertas à interpretação, o que possibilitou identificar os dados das entrevistas de forma subjetiva, dificultando a análise dos resultados com maior precisão. Logo, sugere-se que futuros trabalhos sejam realizados com uma maior amostragem, avaliando o cenário de empresas com áreas de atuação além da indústria aeronáutica, como: montadoras automotivas, indústrias químicas e petroquímicas, cerâmica e vidros, manufatura de pás eólicas, entre outras instituições que gerenciam projetos.

\section{AgRADECIMENTOS}

À empresa ABS e entrevistados pela disposição e aceitação. Ao Prof. Dr. Manoel Gonçales Filho por suportar e revisar esse trabalho com o mais alto critério de qualidade e à minha esposa, Sinara Santana, por apoiar minha vida profissional e acadêmica.

\section{REFERÊNCIAS}

Defense Systems Management College [DSMC]. (2001). System Engineering Fundamentals. Defense Acquisition University Press Fort Belvoir, Virginia, 22060-5565, USA.

Eisenhardt, K. M. (1989). Building Theories from Case Study Research. The Academy of Management Review, 14(4), 532-550. JSTOR. Recuperado de https://www.jstor.org/stable/258557

Fachin, O. (2001). Fundamentos de Metodologia Científica. 3. edição. Editora Atlas, São Paulo, São Paulo, Brasil.

Força Aérea Brasileira [FAB]. (2021). CBA-123 Vector|EMBRAER - FMA. Recuperado de https://www2.fab.mil.br/musal/index.php/aeronaves-em-exposicao/55-avioes/305-vector

Guerra, J. H. L. (2010). Proposta de um Protocolo para o Estudo de Caso em Pesquisas Qualitativas. XXX Encontro Nacional de Engenharia de Produção, 2010, São Carlos, São Paulo, Brasil.

Halligan, R. J. (2021). What is the Wedge Model? Project Performance International (PPI). Recuperado de https://www.ppi-int.com/articles-and-presentations/what-is-the-wedge-model/

Graessler, I., Hentze, J., \& Bruckmann, T. (2018). V-Models for Interdisciplinary Systems Engineering. International Design Conference - Design, 747-756. Paderborn, Detmold, Germany. https://doi.org/10.21278/idc.2018.0333

Lakatos, E. M., \& Marconi, M. A. (2003). Fundamentos de Metodologia Científica. 5a ed. Editora Atlas. São Paulo, São Paulo, Brasil.

Ludke, M., \& André, M. (1986). Pesquisa em Educação: Abordagens Qualitativas. Editora Pedagógica e Universitária. 99p. São Paulo, São Paulo, Brasil. 
Oehmen, J., Thuesen, C., Parraguez, P., \& Geraldi, J. (2015). Complexity Management for Projects, Programmes, and Portfolios: An Engineering Systems Perspective. Project Management Institute, PMI. (PMI White Paper).

Olechowski, A. L., Eppinger, S. D., \& Joglekar, N. (2015). Technology Readiness Levels at 40: A Study of State-of-the-Art Use, Challenges, and Opportunities. MIT Sloan Research Paper, 5127-15. http://dx.doi.org/10.2139/ssrn.2588524

Ottino, J. (2004). Engineering Complex Systems. Nature 427, 399. Northwestern University, Evanston, 60208, Illinois, USA. https://doi.org/10.1038/427399a

Paranhos, L. R. L. (2014). Metodologia da pesquisa aplicada a tecnologia. São Paulo, SENAI-SP Editora, 160p.: il (Série Inovação e Tecnologia. Área Metodologia da Educação).

Project Management Institute [PMI]. (2017). Um Guia do conhecimento em gerenciamento de projetos (Guia PMBOK). 6ed. Global Standard, Newton Square, PA, USA.

Severino, A. J. (2000). Metodologia do trabalho científico. 21a.ed. São Paulo, São Paulo, Brasil. 